\title{
The synthesis of iminothiophenone-fused quinolines and evaluation of their serendipitous reactions
}

\author{
Morteza Shiri, ${ }^{\text {a* }}$ Zeinab Faghihi, ${ }^{\text {a } H o s s e i n ~ A . ~ O s k o u e i, ~}{ }^{a}$ Majid M. Heravi, ${ }^{a}$ Shima Fazelzadeh, ${ }^{\text {a }}$ \\ Behrouz Notash $^{\mathrm{b}}$
}

a Department of Chemistry,Faculty of physics and Chemistry, Alzahra University, Vanak, Tehran 1993893973, Iran. Fax:+982188041344, mshiri@alzahra.ac.ir.

b Department of Chemistry,Faculty of $\backslash$ Chemistry, Shahid Beheshti University, Tehran.

\begin{abstract}
The novel synthesis of tricyclic 2-(cyclohexylimino)thieno[2,3-b]quinolin-3(2H)-ones from the reaction of 2mercaptoquinoline-3-carbaldehydes and isocyanides in methanol without using any additive is described. This protocol proceeds with high atom economy through the formation of $\mathrm{C}-\mathrm{S}$ and $\mathrm{C}-\mathrm{C}$ bond and then oxidation via tandem reaction. Moreover, some other remarkable aspects of this reaction such like the hydrolysis and a three-component reaction with aromatic amine to afford a highly conjugated schiff base is also investigated.
\end{abstract}

Keywords: Aromatic amines, Atom economy, C-C bonds, Isocyanides, Schiff-base, Tandem reaction. Three component reactions. 